\title{
Quasiperiodic boundary conditions for three-dimensional superfluids
}

\author{
Toby S. Wood, ${ }^{*}$ Mae Mesgarnezhad, George W. Stagg, and Carlo F. Barenghi \\ School of Mathematics, Statistics and Physics, Newcastle University, Newcastle upon Tyne, NE1 7RU, United Kingdom
}

(Received 6 February 2019; revised manuscript received 26 June 2019; published 10 July 2019)

\begin{abstract}
We derive boundary conditions that allow a three-dimensional periodic array of superfluid vortices to be modeled in a Cartesian domain. The method is applicable to vortices in the Gross-Pitaevskii description of a superfluid and to fluxtubes in the Ginzburg-Landau description of a superconductor. Unlike standard methods for modeling infinite arrays of vortices, the boundary conditions can be used to study the three-dimensional tangling and reconnection of vortex lines expected in superfluid turbulence. In the two-dimensional case, the boundary conditions include two parameters that determine the lattice offset, which for a single superfluid is essentially arbitrary. In the three-dimensional case the boundary conditions include three parameters that must satisfy a particular linear relationship. We present an algorithm for finding all vortex lattice states within a given domain. We demonstrate the utility of the boundary conditions in two specific problems with imperfect or tangled lattices.
\end{abstract}

DOI: 10.1103/PhysRevB.100.024505

\section{INTRODUCTION}

At sufficiently high densities and low temperatures, many materials transition from a "normal" phase of matter to a quantum superfluid phase. The properties of superfluids are of interest in many laboratory systems, including ultracold atomic gases [1], superconductors [2], and liquid helium [3], and also in neutron stars, which are believed to contain neutron and proton superfluids [4].

The vorticity in a superfluid (and the magnetic flux in a superconductor) is quantized into discrete microscopic filaments called vortices (or fluxtubes in the case of a superconductor). In the Gross-Pitaevskii description, the superfluid is represented by a complex mean-field wave-function $\psi$, called the order parameter, and its density $\rho$ and velocity $\mathbf{v}$ are defined as

$$
\begin{gathered}
\rho=m|\psi|^{2}, \\
\mathbf{v}=\frac{\hbar}{m} \nabla \arg \psi=\frac{\hbar}{m} \nabla \chi,
\end{gathered}
$$

where $\chi \equiv \arg \psi$ is the phase of the superfluid, $m$ is the mass of a superfluid particle (or Cooper pair in a fermionic superfluid), and $\hbar$ is Planck's reduced constant. Equation (2) implies that the superfluid has vanishing vorticity $\nabla \times \mathbf{v}=$ $\mathbf{0}$ at all locations where the phase $\chi$ is smooth. However, vortices can still arise as filaments (in three dimensions) or points (in two dimensions) where $\psi=0$ and hence $\chi$ is undefined. Around a vortex, $\chi$ winds by $2 \pi$, and so each vortex induces a quantum of circulation [5]:

$$
\oint \mathbf{v} \cdot d \mathbf{x}=2 \pi \frac{\hbar}{m}
$$

A superfluid can mimic the rotation of a classical fluid by forming an array of vortices that are, on average, aligned with

*toby.wood@newcastle.ac.uk the rotation axis. The effective macroscopic rotation $\boldsymbol{\Omega}$ of a vortex array is precisely half of its average vorticity, i.e.,

$$
\boldsymbol{\Omega}=\pi \frac{\hbar}{m} \mathbf{n},
$$

where $\mathbf{n}$ represents the number of vortex lines per unit perpendicular cross-sectional area. Although we have here derived Eq. (4) for a Gross-Pitaevskii superfluid, this result—known as Feynman's rule [6] - holds more generally, and has been confirmed in several laboratory experiments with superfluid ${ }^{3} \mathrm{He}$ and ${ }^{4} \mathrm{He}$ [7-9]. Equation (4) has a direct analogy in a (nonrotating) superconductor; in that case, the magnetic flux is quantized, and the macroscopic magnetic field is proportional to the number of fluxtubes per unit area. Given the close analogy between the behavior of vortices and fluxtubes, for brevity from here on we will use the term vortices to refer to both cases collectively.

In most superfluids, and in type-II superconductors, the vortices are mutually repulsive. When their number density is sufficiently high, they therefore tend to form a periodic array, which we call a lattice (see Fig. 1). A lattice is characterized by a pair of primitive lattice vectors $\ell_{1}$ and $\ell_{2}$ from which the vortex density can be calculated using the formula $\mathbf{n}=$ $\frac{\ell_{1} \times \ell_{2}}{\left|\ell_{1} \times \ell_{2}\right|^{2}}$. Many superfluid systems contain a large number of vortices - too many for all of them to be modeled individually. For example, in a typical liquid helium experiment with a cross-sectional area of $1 \mathrm{~cm}^{2}$, rotating at a rate of $1 \mathrm{rad} / \mathrm{s}$, there are approximately 2000 vortices. A more extreme example is the outer core of a typical neutron star, which is believed to contain of order $10^{16}$ vortices and $10^{30}$ fluxtubes [10,11], within a radius of $10 \mathrm{~km}$. To model such systems, it is natural to consider a small but representative piece, and impose periodic boundary conditions, formally regarding the system as having infinite extent. However, in a vortex lattice the phase of the superfluid is not periodic (see Fig. 1); rather, it is quasiperiodic, in a sense that we define in the next section. 


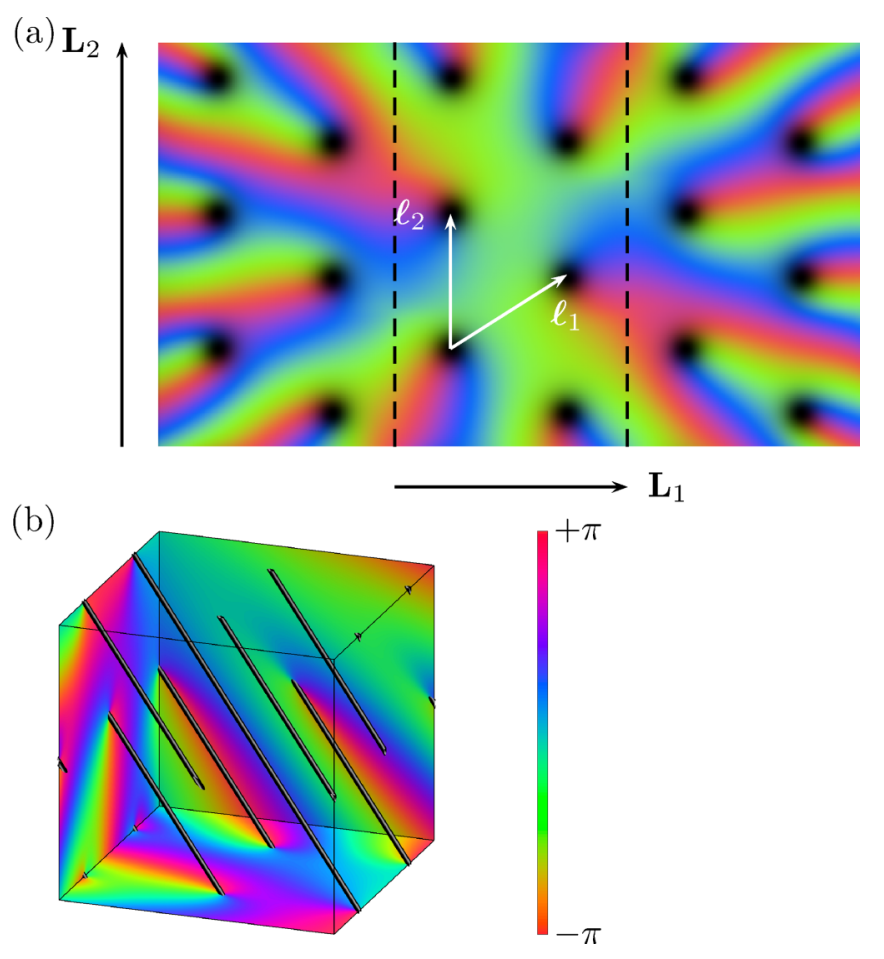

FIG. 1. (a) The order parameter $\psi$ for a hexagonal lattice of vortices (black circles), with primitive vectors $\ell_{1}$ and $\ell_{2}$. The color represents the phase of the superfluid $\chi$, and the brightness represents its density $|\psi|^{2}$, and hence the vortex cores appear black. The vectors $\mathbf{L}_{1}$ and $\mathbf{L}_{2}$ indicate the sides of the computational domain, which is plotted alongside two quasiperiodic copies. The density is spatially periodic, but the phase is not. (b) The phase of an inclined rectangular lattice in a cube. The vortex lines (drawn in black) are arranged periodically within the domain.

The traditional method for modeling an infinite array of vortices is to use an analytical approximation for the order parameter-the lowest Landau level $[12,13]$. This approach is well suited to superfluids that are essentially two dimensional, and particularly for a tightly packed lattice, for which the analytical approximation holds to high accuracy. However, this method cannot be applied to tangled vortex lines, or to superfluids with large density variations.

An alternative method is to model a representative piece of the system, and apply quasiperiodic boundary conditions that relate the value of the order parameter on opposite sides of the domain. The two-dimensional (2D) case (in which the computational domain is a perpendicular cross section through the vortex array) has recently been considered by Mingarelli et al. [14,15], but the fully three-dimensional (3D) case presents additional challenges. Obtaining suitable 3D boundary conditions is necessary to describe two-component systems in which the arrays are mutually inclined, as is the case for the vortex and fluxtube arrays in the core of a neutron star, for example [11,16].

The plan for this paper is as follows. In Sec. II we describe the essential properties of vortex and fluxtube arrays, and deduce the general form of the quasiperiodic boundary conditions in 2D. In Sec. III we verify explicitly that these boundary conditions are compatible with all possible 2D lattice configurations. In Sec. IV we generalize our results to $3 \mathrm{D}$, obtaining quasiperiodic boundary conditions for an inclined array in a cuboidal domain. Two particular applications of these boundary conditions are presented in Sec. V, demonstrating that a periodic lattice is not the preferred state in all cases. We conclude in Sec. VI by discussing the implications for systems of many vortices.

\section{SYMMETRY PROPERTIES OF VORTEX AND FLUXTUBE ARRAYS}

Consider an infinite array of vortices with mean angular velocity $\boldsymbol{\Omega}$. Suppose that the vector $\mathbf{L}$ represents a discrete translational symmetry of the array, i.e., that the vortex locations are unchanged under a coordinate shift $\mathbf{x} \rightarrow \mathbf{x}+\mathbf{L}$, where $\mathbf{x}$ is the position vector. In that case the relative velocity of the superfluid (that is, the velocity in the rotating frame) must be periodic, i.e.,

$$
\begin{aligned}
\left.\frac{\hbar}{m} \nabla \chi\right|_{\mathbf{x}+\mathbf{L}}-\mathbf{\Omega} \times(\mathbf{x}+\mathbf{L}) & =\left.\frac{\hbar}{m} \nabla \chi\right|_{\mathbf{x}}-\mathbf{\Omega} \times \mathbf{x} \\
\left.\Rightarrow \boldsymbol{\nabla} \chi\right|_{\mathbf{x}} ^{\mathbf{x}+\mathbf{L}} & =\frac{m}{\hbar} \mathbf{\Omega} \times \mathbf{L} \\
& =\pi \mathbf{n} \times \mathbf{L} \\
\left.\Rightarrow \chi\right|_{\mathbf{x}} ^{\mathbf{x}+\mathbf{L}} & =\pi(\mathbf{n} \times \mathbf{L}) \cdot \mathbf{x}+c \bmod 2 \pi,
\end{aligned}
$$

where $c$ is some unknown constant, whose value is defined only modulo $2 \pi$. A similar line of reasoning can be applied to an array of magnetic fluxtubes in a superconductor, with magnetic vector potential $\mathbf{A}$. In this system, the definition of the superfluid velocity is modified in accordance with minimal coupling:

$$
\mathbf{v}=\frac{\hbar}{m} \nabla \chi-\frac{q}{m c} \mathbf{A},
$$

where $q$ is the electric charge of a superfluid particle (or Cooper pair). If we choose to work in a symmetric gauge, then we have $\mathbf{A}=\frac{1}{2} \mathbf{H} \times \mathbf{x}+\mathbf{A}^{\prime}$, where $\mathbf{H}$ is the mean magnetic field, and $\mathbf{A}^{\prime}$ is a periodic perturbation. In that case, we can repeat the above argument with $\boldsymbol{\Omega}$ replaced by $\frac{q}{2 m c} \mathbf{H}$, once again arriving at Eq. (5), with $\mathbf{n}$ now representing the number of fluxtubes per unit area. From here on we will therefore adopt a symmetric gauge whenever referring to the phase in a superconductor, although of course all of our results can be easily transformed into any gauge.

Equation (5) defines precisely the sense in which $\chi$ is quasiperiodic, and this is the fundamental result for obtaining boundary conditions for numerical calculations. In a typical calculation, the domain will be a Cartesian box, as illustrated for example in Fig. 1(a), whose sides are the vectors $\mathbf{L}_{1}$ and $\mathbf{L}_{2}$. (For the moment we will only consider a two-dimensional box, and the three-dimensional box will be considered later, in Sec. IV.) Note, however, that the vortices within the domain need not form a periodic lattice; we assume only that the arrangement of vortices within the domain is duplicated in each of its quasiperiodic neighbors. In that case the number of vortices per unit area can be calculated as

$$
\mathbf{n}=\frac{N}{L_{1} L_{2}} \mathbf{e}_{3},
$$


where $N$ is the number of vortices within the computational domain, which must be an integer. More precisely, $N$ is the total quanta of vortices in the direction $\mathbf{e}_{3}$; if the domain contains $N^{+}$positive (anticlockwise) and $N^{-}$negative (clockwise) vortices, then $N=N^{+}-N^{-}$. We observe that the sides of the computational domain $\mathbf{L}_{1}$ and $\mathbf{L}_{2}$ must be translational symmetries of the vortex array, even if the vortices within the domain do not form a lattice. Equation (5) can therefore be used to obtain a pair of quasiperiodic boundary conditions:

$$
\left.\chi\right|_{\mathbf{x}} ^{\mathbf{x}+\mathbf{L}_{i}}=\pi\left(\mathbf{n} \times \mathbf{L}_{i}\right) \cdot \mathbf{x}+c_{i} \bmod 2 \pi, \quad i=1,2 .
$$

It is not immediately clear whether these boundary conditions are mutually compatible, i.e., whether they can be imposed without producing corner singularities in the phase of the superfluid. If we follow a circuit anticlockwise around the edge of the computational domain, the boundary conditions (8) imply that we experience a net phase shift:

$$
\begin{aligned}
& \left.\chi\right|_{\mathbf{x}} ^{\mathbf{x}+\mathbf{L}_{1}}+\left.\chi\right|_{\mathbf{x}+\mathbf{L}_{1}} ^{\mathbf{x}+\mathbf{L}_{1}+\mathbf{L}_{2}}+\left.\chi\right|_{\mathbf{x}+\mathbf{L}_{1}+\mathbf{L}_{2}} ^{\mathbf{x}+\mathbf{L}_{2}}+\left.\chi\right|_{\mathbf{x}+\mathbf{L}_{2}} ^{\mathbf{x}} \\
& \quad=-2 \pi \mathbf{n} \cdot\left(\mathbf{L}_{1} \times \mathbf{L}_{2}\right)=-2 \pi N \quad \bmod 2 \pi .
\end{aligned}
$$

Hence these boundary conditions are mutually compatible provided that $N$ is an integer.

The boundary conditions (8) contain a pair of unknown constants $c_{1}$ and $c_{2}$, whose values must be specified prior to performing any numerical simulation. Recently, Mingarelli et al. $[14,15]$ have used these boundary conditions to simulate infinite vortex arrays in a 2D Cartesian domain, and have shown that changing the values of $c_{1}$ and $c_{2}$ corresponds to a spatial translation of the vortex array. The main motivation for the present work is to precisely relate the values of the parameters $c_{1}, c_{2}$ to the vortex locations in the lattice, and to generalize the quasiperiodic boundary conditions to the fully 3D case of an inclined vortex array, as illustrated in Fig. 1(b).

\section{THE VELOCITY FIELD OF A VORTEX LATTICE}

To elucidate the physical significance of the parameters $c_{1}$ and $c_{2}$ in Eq. (8), we consider the case of a periodic vortex lattice, for which the phase can be expressed analytically [17]. Suppose we have an infinite lattice of point vortices in the complex $z$ plane, at the locations

$$
z=z_{0}+P \pi+Q \pi \tau, \quad \text { for } P, Q \in \mathbb{Z},
$$

where $z_{0}$ and $\tau$ are some complex constants. If the lattice is uniformly rotating anticlockwise about the origin, then the phase of the superfluid must be (apart from an arbitrary constant)

$$
\chi=\operatorname{Im}\left\{\frac{\left(z-2 i \operatorname{Im}\left\{z_{0}\right\}\right)^{2}}{2 \pi \operatorname{Im}\{\tau\}}+\ln \vartheta_{1}\left(z-z_{0} ; \tau\right)\right\},
$$

where $\vartheta_{1}$ is the Jacobi theta function. Using the well-known quasiperiodicity properties of $\vartheta_{1}$, we can thus evaluate the phase shift between an arbitrary point $z$ and any of its images $z+P \pi+Q \pi \tau$

$$
\left.\chi\right|_{z} ^{z+P \pi+Q \pi \tau}=\frac{\operatorname{Im}\left\{\left(P+Q \tau^{\star}\right)\left(z-2 z_{0}\right)\right\}}{\operatorname{Im}\{\tau\}}+\pi(P+Q+P Q),
$$

where $\tau^{\star}$ is the complex conjugate of $\tau$. The same result can be expressed in vector form as

$$
\begin{aligned}
\left.\chi\right|_{\mathbf{x}} ^{\mathbf{x}+P \ell_{1}+Q \ell_{2}}= & \pi \mathbf{n} \cdot\left[\left(P \ell_{1}+Q \ell_{2}\right) \times\left(\mathbf{x}-2 \mathbf{x}_{0}\right)\right] \\
& +\pi(P+Q+P Q),
\end{aligned}
$$

where $\ell_{1}$ and $\ell_{2}$ are the primitive vectors of the vortex lattice, and where $\mathbf{x}_{0}$ is the location of any vortex in the lattice. We will refer to $\mathbf{x}_{0}$ as the lattice offset. Although we have derived this result assuming that the vortex locations are given by Eq. (9), we note that the phase of the superfluid must be invariant under a rotation about the origin or a uniform rescaling of the axes. Since any vortex lattice can be put into the form of Eq. (9) under an appropriate rotation and rescaling, the result (12) therefore applies to all lattices.

If we have a vortex lattice in a rectangular domain with sides $\mathbf{L}_{1}$ and $\mathbf{L}_{2}$, as in Fig. 1(a) for example, then we must have $\mathbf{L}_{i}=P_{i} \ell_{1}+Q_{i} \ell_{2}$ for some integers $P_{i}, Q_{i}$. Using Eq. (12) we deduce that the unknown constants in the boundary conditions (8) are related to the lattice offset $\mathbf{x}_{0}$ :

$$
c_{i}=-2 \pi\left(\mathbf{n} \times \mathbf{L}_{i}\right) \cdot \mathbf{x}_{0}+\pi\left(P_{i}+Q_{i}+P_{i} Q_{i}\right) \bmod 2 \pi .
$$

Crucially, for any given lattice and any choice of $c_{1}, c_{2}$, this equation can always be solved for $\mathbf{x}_{0}$. Therefore the choice of constants $c_{i}$ is essentially arbitrary; their values only determine the lattice offset $\mathbf{x}_{0}$, and do not constrain the shape of the lattice. The geometrical meaning of $c_{i}$ can be made clearer by rewriting Eq. (13) as

$$
c_{i}=-2 \pi\left(\mathbf{n} \times \mathbf{L}_{i}\right) \cdot \overline{\mathbf{x}}+\pi N \bmod 2 \pi,
$$

where $\overline{\mathbf{x}}$ is the average location of the $N$ vortices in the domain, modulo $\left(L_{1} / N, L_{2} / N\right)$ (see the Appendix).

In summary, then, to model a $2 \mathrm{D}$ array of vortices in a domain with sides $\mathbf{L}_{1}$ and $\mathbf{L}_{2}$, we can use the boundary conditions (8), and the values of $c_{i}$ merely serve to fix the average position of the vortices.

\section{BOUNDARY CONDITIONS IN 3D}

So far we have only considered $2 \mathrm{D}$ vortex arrays, i.e., arrays of point vortices. Our results are easily extended to $3 \mathrm{D}$ vortex arrays that are, on average, aligned in the direction of the third coordinate, i.e., $\mathbf{n} \| \mathbf{e}_{3}$. In that case, we have quasiperiodic boundary conditions in the first two coordinates, given by Eq. (8), and a periodic boundary condition in the third coordinate. As in the 2D case, the boundary conditions include two parameters $c_{1}$ and $c_{2}$, whose values control the transverse offset of the array.

However, there are situations in which the vortex array cannot be assumed to align with any of the coordinates. The core of a neutron star is one example, in which there are arrays of vortices and fluxtubes whose mean directions align with the star's rotation and magnetic axes, respectively, which in general are mutually inclined [18] (as shown schematically in Fig. 2). To model an inclined vortex array, in a Cartesian box whose edges are $\mathbf{L}_{1}, \mathbf{L}_{2}$, and $\mathbf{L}_{3}$, we must apply quasiperiodic boundary conditions in all three directions. Since each of the vectors $\mathbf{L}_{i}$ represents a translational symmetry of the array, these boundary conditions must have the form

$$
\left.\chi\right|_{\mathbf{x}} ^{\mathbf{x}+\mathbf{L}_{i}}=\pi\left(\mathbf{n} \times \mathbf{L}_{i}\right) \cdot \mathbf{x}+c_{i} \bmod 2 \pi, \quad i=1,2,3 .
$$




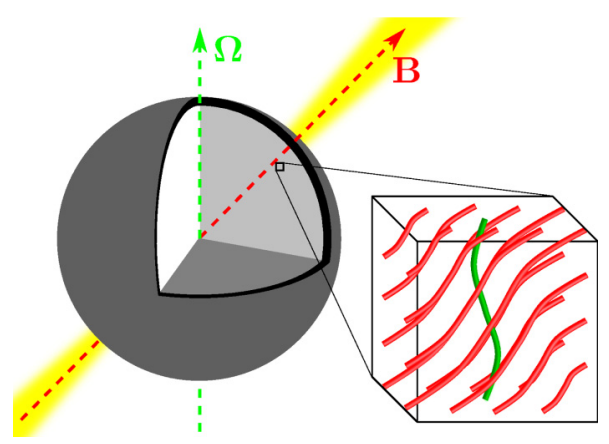

FIG. 2. Illustration of the internal structure of a neutron star. The dashed green and red lines indicate the rotation and magnetic axes, respectively. Below the star's crust (shown in black) the neutrons and protons form superfluids. The vorticity of the neutron fluid is quantized into vortices (shown in green) that on average align with the star's rotation axis. The magnetic field of the proton fluid is quantized into fluxtubes (shown in red) that on average align with the star's macroscopic magnetic field.

For these boundary conditions to be mutually compatible, each face of the domain must be intersected by an integer number of vortices, in which case we have

$$
\mathbf{n}=\left(\frac{N_{1}}{L_{2} L_{3}}, \frac{N_{2}}{L_{3} L_{1}}, \frac{N_{3}}{L_{1} L_{2}}\right),
$$

where the vector $\mathbf{N}=\left(N_{1}, N_{2}, N_{3}\right) \in \mathbb{Z}^{3}$ counts the number of intersections. Our boundary conditions therefore contain three parameters $c_{i}$, but by analogy with the aligned array case described above, we anticipate that only two of these parameters are independent. Choosing their values arbitrarily would impose a nonzero phase shift, and therefore by Eq. (2) a flow, parallel to the vortex array. To avoid imposing such a flow, we must choose the values of $c_{1}, c_{2}, c_{3}$ such that

$$
\left.\chi\right|_{\mathbf{x}} ^{\mathbf{x}+\mathbf{L}}=0 \bmod 2 \pi
$$

for any translational symmetry vector $\mathbf{L}$ that is parallel to $\mathbf{n}$. One such symmetry vector is

$$
\mathbf{L}_{\|} \equiv\left(\frac{N_{1}}{G} L_{1}, \frac{N_{2}}{G} L_{2}, \frac{N_{3}}{G} L_{3}\right)=\frac{L_{1} L_{2} L_{3}}{G} \mathbf{n},
$$

where $G \equiv \operatorname{gcd}\{\mathbf{N}\}$ is the greatest common positive divisor of $N_{1}, N_{2}, N_{3}$. When the condition (17) is applied for the symmetry vector $\mathbf{L}_{\|}$, using the boundary conditions (15), it implies the following relation between the parameters $c_{i}$ :

$$
\mathbf{N} \cdot \mathbf{c}=\pi N_{1} N_{2} N_{3} / G \bmod 2 \pi G .
$$

Furthermore, using the fact that the quantity $\left(N_{1} / G\right)\left(N_{2} / G\right)\left(N_{3} / G\right)(G-1) G$ is guaranteed to be an even integer, we can write this result in the simpler but equivalent form

$$
\mathbf{N} \cdot \mathbf{c}=\pi N_{1} N_{2} N_{3} \quad \bmod 2 \pi G .
$$

It is clear that this condition is necessary to avoid imposing a flow along the vortex array, and in fact it is also sufficient, i.e., provided that this condition holds, it is always possible to find a uniformly rotating vortex lattice that satisfies our boundary conditions and has zero flow along the rotation axis. To demonstrate the sufficiency of condition (20), we can simply observe that each of the $c_{i}$ is only defined modulo $2 \pi$, and therefore the value of $\mathbf{N} \cdot \mathbf{c}$ cannot be imposed to higher accuracy than given by Eq. (20). This is essentially Bézout's identity.

In order to prove the sufficiency of condition (20) more explicitly, in the Appendix we present an algorithm for generating all possible lattice states, for a given choice of $\mathbf{N} \in$ $\mathbb{Z}^{3}$, and demonstrate that each lattice is compatible with the boundary conditions (15) if condition (20) holds.

\section{APPLICATIONS}

To demonstrate the utility of our quasiperiodic boundary conditions, in the following sections we consider two specific applications.

\section{A. Imperfections in a 2D fluxtube lattice}

We consider a two-dimensional Ginzburg-Landau superconductor in the symmetric Weyl gauge. This system is characterized by the complex order parameter $\psi\left(x_{1}, x_{2}, t\right)$ and the magnetic vector potential $\mathbf{A}\left(x_{1}, x_{2}, t\right)$. The free energy, in dimensionless units, is

$\mathcal{H}=\int\left[\frac{1}{2}\left(|\psi|^{2}-1\right)^{2}+|(\nabla-i \mathbf{A}) \psi|^{2}+\kappa^{2}|\nabla \times \mathbf{A}|^{2}\right] d^{2} \mathbf{x}$, where $\kappa$ is the Ginzburg-Landau parameter. Provided that $\kappa>1 / \sqrt{2}$, the lowest energy state in this system is generally achieved by quantizing the magnetic field $\mathbf{B}=\boldsymbol{\nabla} \times \mathbf{A}$ into discrete fluxtubes, i.e., we have a type-II superconductor. Moreover, in the absence of geometric constraints the fluxtubes preferentially form a hexagonal lattice $[12,19]$. In the dissipative regime appropriate for laboratory superconductors, $\psi$ and A evolve according to the time-dependent GinzburgLandau equations

$$
-\frac{\partial \psi}{\partial t}=\frac{\delta \mathcal{H}}{\delta \psi^{\star}} \quad \text { and } \quad-\frac{2 \kappa^{2}}{\eta} \frac{\partial \mathbf{A}}{\partial t}=\frac{\delta \mathcal{H}}{\delta \mathbf{A}},
$$

where $\eta$ is the dimensionless resistivity. These equations guarantee that the free energy decreases monotonically, until a local minimum is achieved.

We solve these equations using a discrete approximation to the free energy, in which the components of $\mathbf{A}$ are defined on the links between the gridpoints for $\psi$. The gauge coupling between $\psi$ and $\mathbf{A}$ is implemented using a standard Peierls substitution, i.e., by making the approximation

$$
\begin{aligned}
& \int\left|\left(\partial_{i}-i A_{i}\right) \psi\right|^{2} d^{2} \mathbf{x} \\
& \simeq \sum_{\mathbf{x}}\left|\frac{\left.\psi\right|_{\mathbf{x}+\delta x_{i} \mathbf{e}_{i}}-\left.\psi\right|_{\mathbf{x}} \exp \left(\left.i \delta x_{i} A_{i}\right|_{\mathbf{x}+\frac{1}{2} \delta x_{i} \mathbf{e}_{i}}\right)}{\delta x_{i}}\right|^{2} \delta x_{1} \delta x_{2},
\end{aligned}
$$

where $\delta x_{i}$ is the grid spacing and where the sum is taken over the gridpoints for $\psi$.

Our goal is to simulate a periodic array of fluxtubes, with $N$ fluxtubes in the computational domain, which is a rectangle with sides $L_{1}$ and $L_{2}$. We therefore define $\mathbf{n}=\frac{N}{L_{1} L_{2}} \mathbf{e}_{3}$, impose periodic boundary conditions for 


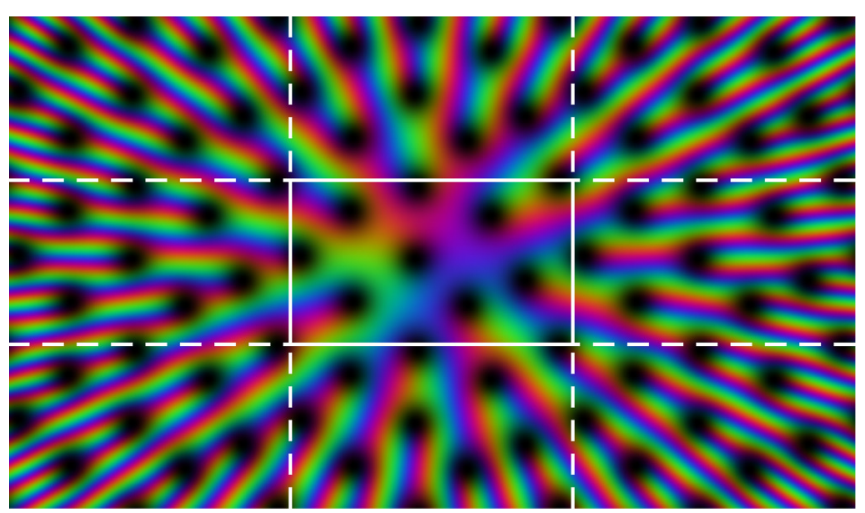

FIG. 3. The ground state for a system of $N=9$ fluxtubes in a domain of size $(10 \sqrt{3}, 10)$, indicated as a white rectangle. The color scheme matches that in Fig. 1(a). The computational domain is plotted alongside its eight nearest quasiperiodic copies. For this domain size, the ground state is not a lattice.

$\mathbf{A}^{\prime}=\mathbf{A}-\pi \mathbf{n} \times \mathbf{x}$, and impose the quasiperiodic boundary conditions (8) for $\psi$. In the results presented below we have taken the constants in these boundary conditions to be $c_{1}=$ $c_{2}=\pi N$. We have also set $\kappa=5$ and $\eta=1$, and used a rectangular computational domain of size $L_{1}=10 \sqrt{3}, L_{2}=$ 10. For a domain of this size and aspect ratio, a hexagonal lattice of fluxtubes can be achieved with $N=2,6,8,18$, or 24. (For $N \gtrsim 27$, the mean magnetic field $\mathbf{H}=2 \pi \mathbf{n}$ exceeds the upper critical field, which is unity in our dimensionless units, and superconductivity is lost.) We have set $N=9$, in order to study the ground state in a case where the preferred symmetry is frustrated by the boundary conditions. In the case $N=9$ there are $\sigma(9)=13$ possible lattice configurations (see the Appendix), only two of which are local minima of the free energy, and hence linearly stable. In order to identify the true ground state for this system, we have used a range of initial conditions, including each of the 13 periodic lattice states, and states with fluxtubes at randomly chosen locations.

For most choices of initial conditions, we find that the fluxtubes do not ever achieve a lattice configuration. Instead they reach a steady state with an irregular but roughly uniform spacing, as illustrated in Fig. 3. In this state, the free energy was computed to be $\mathcal{H}=514.063$, which is smaller than the free energy in both of the stable lattice states (which have $\mathcal{H}=514.103)$, demonstrating that the ground state for the chosen domain size is not a lattice. (Of course the average free energy per unit area in Fig. 3 is still larger than that for a hexagonal lattice with the same mean magnetic field, which would be the preferred state in the absence of geometric constraints.)

\section{B. Tangling in a 3D vortex array}

We consider a single-component superfluid in the rotating frame. We include a potential whose gradient exactly balances the centrifugal force, so that the effective potential vanishes in the rotating frame, allowing the bulk density of the superfluid to be homogeneous. The free energy in the Gross-Pitaevskii

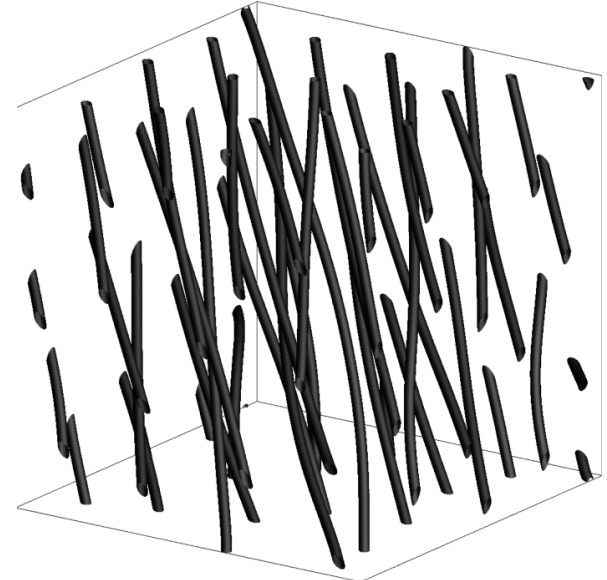

FIG. 4. A local minimum of the free energy (23) for a cube of side $L=64$, each face of which is intersected by 16 vortices. The vortex lines (drawn in black) remain tangled, and do not form a lattice.

description can then be written in dimensionless form as

$$
\mathcal{H}=\int\left[\frac{1}{2}\left(|\psi|^{2}-1\right)^{2}+|\nabla \psi-i \pi \psi \mathbf{n} \times \mathbf{x}|^{2}\right] d^{3} \mathbf{x},
$$

where $\pi \mathbf{n}$ is the dimensionless angular velocity of the frame of reference. In our numerical calculations, the free energy is approximated using a discretization similar to that described in the previous section.

We choose the computational domain to be a cube of side $L=64$, and we take $\mathbf{n}$ to be

$$
\mathbf{n}=\left(\frac{16}{L^{2}}, \frac{16}{L^{2}}, \frac{16}{L^{2}}\right) .
$$

We impose the boundary conditions (15) for $\psi$, with $c_{1}=$ $c_{2}=c_{3}=0$, which satisfies Eq. (20) in this case.

Starting from a random initial condition, we solve the imaginary-time Gross-Pitaevskii equation

$$
-\frac{\partial \psi}{\partial t}=\frac{\delta \mathcal{H}}{\delta \psi^{\star}},
$$

which guarantees that the free energy decreases monotonically. We anticipate that the true ground state for this system will be a periodic and rectilinear array of vortices. However, as illustrated in Fig. 4, in most of our simulations the vortex array never achieves such a state, and instead converges to a metastable state in which the vortex lines remain somewhat tangled. We find that multiple metastable states exist with various degrees of tangling, demonstrating that the vortex array is a "glassy" system, in spite of the long-range order imposed by the quasiperiodic boundary conditions. In order for the system to reach the true ground state, further reconnection of the vortex lines would need to be induced through an appropriate injection of energy (i.e., annealing). This can be achieved by reducing the size of the domain, and thereby forcing the tangled vortex lines closer together. In our simulations, we find that such reconnections occur once the distance between vortex lines becomes comparable to the size of the vortex core, which is order unity in our dimensionless 
units. In the case shown in Fig. 4, reducing the size of the domain to $L=48$ is sufficient to guarantee that the vortex lines completely untangle.

\section{CONCLUSIONS}

We have derived quasiperiodic boundary conditions for an inclined vortex lattice in a Cartesian domain with sides $\mathbf{L}_{1}, \mathbf{L}_{2}, \mathbf{L}_{3}$. The most general boundary conditions are given by Eq. (15), where $\mathbf{n}=\left(\frac{N_{1}}{L_{2} L_{3}}, \frac{N_{2}}{L_{3} L_{1}}, \frac{N_{3}}{L_{1} L_{2}}\right)$ is the vortex density, the integers $N_{i}$ count the number of intersections of the lattice with the faces of the domain, and the $c_{i}$ parameters must satisfy condition (20).

Our boundary conditions contain two free parameters, whose values determine the lattice offset, relative to the rotation axis. In the case of a single homogeneous superfluid, these parameters are essentially arbitrary, but for multicomponent superfluids they determine the relative displacement between the lattices, which can be crucial for the dynamics [15]. Importantly, the choice of these parameters does not affect the symmetry of the lattice, which is determined entirely by the domain size and by $\mathbf{N}=\left(N_{1}, N_{2}, N_{3}\right)$. For given $\mathbf{N} \in$ $\mathbb{Z}^{3}$ the number of distinct lattice states is given by the sum of all positive common divisors of $\left\{N_{1}, N_{2}, N_{3}\right\}$. In the particular case $\mathbf{N}=(0,0, N)$ this reduces to a well-known result in lattice algebra [20].

The results of Sec. V demonstrate two situations in which a perfect lattice is not achieved, despite the long-range order imposed by the boundary conditions. In the first case, the dimensions of the domain $L_{1}, L_{2}$ frustrate the preferred hexagonal symmetry, and the ground state is not a lattice at all. In the second case, the vortex topology depends on the initial conditions for the simulation, indicating that there are many (meta)stable vortex configurations.

Although the simulations we have presented include only a relatively small number of vortices, we expect that these results will hold also for larger-scale systems, such as neutron stars. In fact, as the size of the system increases so does the number of possible vortex configurations, and also the number of metastable states. As an example, we have repeated the simulation shown in Fig. 3 with a domain of twice the size in both directions, and with the same density of vortices $(N=36)$. This system has a much larger number of possible lattice states $[\sigma(36)=91]$, and we have also identified several metastable nonlattice states, one of which is illustrated in Fig. 5. All of the stable states in this system roughly approximate a hexagonal lattice within most of the domain, and all have a lower free energy per unit area than the ground state shown in Fig. 3. In general, as the size of the system increases the vortex arrangement becomes less constrained by the domain aspect ratio, but the likelihood of becoming trapped in a metastable state increases.

In the case of neutron stars, it is well known that the time required to achieve the true ground state globally is much longer than the age of the system [4]. However, it is often assumed that the arrays of vortices and fluxtubes are roughly periodic and rectilinear on scales comparable to the vortex separation. Our results suggest that, even on this scale, the vortex arrays are likely to be aperiodic and tangled. However, determining the true nature of these vortices would require a

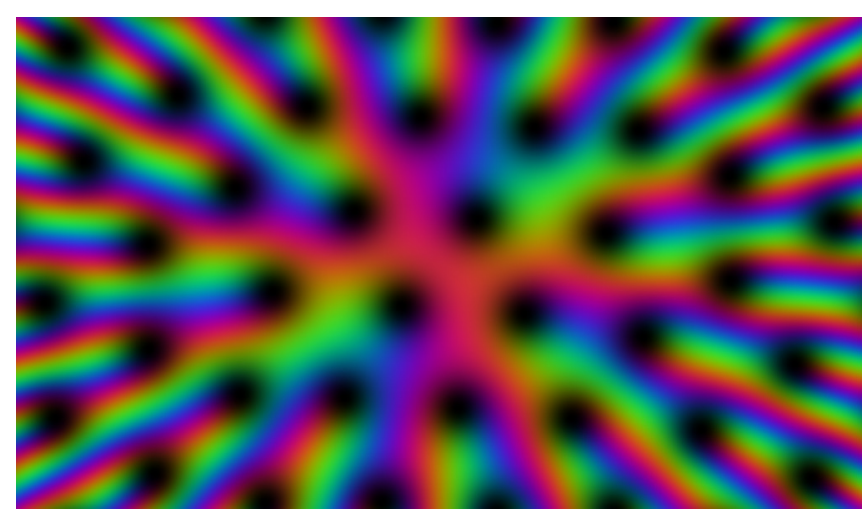

FIG. 5. A metastable state with $N=36$ fluxtubes in a domain of size $(20 \sqrt{3}, 20)$. Other parameters are as in Fig. 3.

more complete model of neutron star matter, which is beyond the scope of this paper.

\section{ACKNOWLEDGMENTS}

We thank Tom Billam, Vanessa Graber, James Waldron, and the anonymous referees, for helpful comments and discussions. C.F.B.'s initial work on this problem was funded by STFC Grant ST/K001019/1, and part of T.S.W.'s time was funded by EPSRC Grant EP/R024952/1.

\section{APPENDIX: EXPLICIT LATTICE CONSTRUCTION}

Each periodic vortex lattice is characterised by a pair of primitive vectors $\ell_{1}$ and $\ell_{2}$, and its offset, that is, the separation between the rotation axis and the closest vortex. Our goal is to identify all possible pairs $\boldsymbol{\ell}_{1}$ and $\boldsymbol{\ell}_{2}$ for a given choice of $\mathbf{L}=\left(L_{1}, L_{2}, L_{3}\right) \in \mathbb{R}^{3}$ and $\mathbf{N}=\left(N_{1}, N_{2}, N_{3}\right) \in \mathbb{Z}^{3}$, which represent the size of the Cartesian domain and the number of lattice intersections with each face. Each pair $\ell_{1}, \ell_{2}$ must satisfy the equation

$$
\mathbf{n}=\left(\frac{N_{1}}{L_{2} L_{3}}, \frac{N_{2}}{L_{3} L_{1}}, \frac{N_{3}}{L_{1} L_{2}}\right)=\frac{\boldsymbol{\ell}_{1} \times \boldsymbol{\ell}_{2}}{\left|\ell_{1} \times \boldsymbol{\ell}_{2}\right|^{2}},
$$

and moreover each side of the domain $\mathbf{L}_{i}$ must be a translational symmetry of the lattice. So if we define $\tilde{\mathbf{L}}_{i}$ to be the projection of $\mathbf{L}_{i}$ in the plane perpendicular to $\mathbf{n}$, then we must have

$$
\tilde{\mathbf{L}}_{i} \equiv \mathbf{L}_{i}-\frac{\mathbf{L}_{i} \cdot \mathbf{n}}{|\mathbf{n}|^{2}} \mathbf{n}=P_{i} \ell_{1}+Q_{i} \ell_{2}
$$

for some $P_{i}, Q_{i} \in \mathbb{Z}$. The parallelogram with sides $\tilde{\mathbf{L}}_{i}, \tilde{\mathbf{L}}_{j}$ is intersected by the same number of vortices as is the rectangle with sides $\mathbf{L}_{i}, \mathbf{L}_{j}$, and so

$$
\begin{aligned}
\tilde{\mathbf{L}}_{1} \times \tilde{\mathbf{L}}_{2} & =N_{3} \boldsymbol{\ell}_{1} \times \boldsymbol{\ell}_{2}, \\
\tilde{\mathbf{L}}_{2} \times \tilde{\mathbf{L}}_{3} & =N_{1} \boldsymbol{\ell}_{1} \times \boldsymbol{\ell}_{2}, \\
\tilde{\mathbf{L}}_{3} \times \tilde{\mathbf{L}}_{1} & =N_{2} \boldsymbol{\ell}_{1} \times \boldsymbol{\ell}_{2} .
\end{aligned}
$$

These relations imply that the vectors $\mathbf{P}=\left(P_{1}, P_{2}, P_{3}\right)$ and $\mathbf{Q}=\left(Q_{1}, Q_{2}, Q_{3}\right)$ must satisfy the equation

$$
\mathbf{P} \times \mathbf{Q}=\mathbf{N} \text {. }
$$




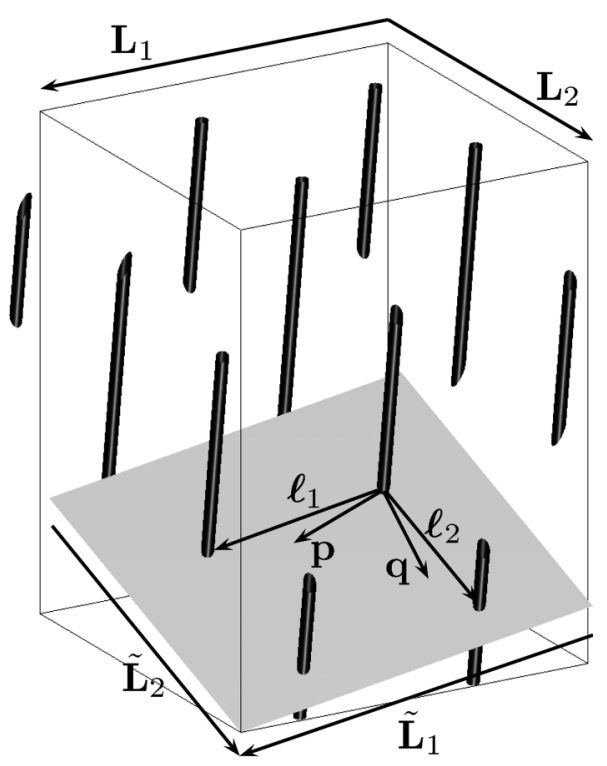

FIG. 6. The vectors $\tilde{\mathbf{L}}_{1}$ and $\tilde{\mathbf{L}}_{2}$ are the projections of $\mathbf{L}_{1}$ and $\mathbf{L}_{2}$ in the plane perpendicular to the vortex array. In this plane, the vortex locations are defined by the primitive lattice vectors $\boldsymbol{\ell}_{1}$ and $\boldsymbol{\ell}_{2}$, and the vectors $\mathbf{p}$ and $\mathbf{q}$ define the reciprocal lattice.

Finding all lattice configurations is therefore equivalent to finding all pairs of vectors $\mathbf{P}, \mathbf{Q} \in \mathbb{Z}^{3}$ that satisfy Eq. (A3). Indeed, once $\mathbf{P}$ and $\mathbf{Q}$ are known, we can invert Eq. (A2) to obtain explicit expressions for $\ell_{1}$ and $\ell_{2}$ :

$$
\begin{gathered}
\ell_{1}=\frac{\mathbf{q} \times \mathbf{n}}{|\mathbf{n}|^{2}} \quad \text { and } \ell_{2}=\frac{\mathbf{n} \times \mathbf{p}}{|\mathbf{n}|^{2}}, \\
\text { where } \mathbf{p} \equiv\left(\frac{P_{1}}{L_{1}}, \frac{P_{2}}{L_{2}}, \frac{P_{3}}{L_{3}}\right) \text { and } \mathbf{q} \equiv\left(\frac{Q_{1}}{L_{1}}, \frac{Q_{2}}{L_{2}}, \frac{Q_{3}}{L_{3}}\right) \text {. }
\end{gathered}
$$

Equation (A3) implies that $\mathbf{p} \times \mathbf{q}=\mathbf{n}$, which together with Eq. (A4) demonstrates that the vectors $\mathbf{p}, \mathbf{q}$ define the reciprocal of the vortex lattice [21]. The relations between the various vector quantities are illustrated in Fig. 6.

For each lattice, i.e., for each solution of Eq. (A3), we can calculate the three phase shifts $\left.\chi\right|_{\mathbf{x}} ^{\mathbf{x}+\mathbf{L}_{i}}$ from Eq. (12). As in the case of a 2D lattice, we deduce that the unknown constants $c_{i}$ in our quasiperiodic boundary conditions (15) are given by Eq. (13), with $i=1,2,3$. However, unlike the 2D case, in general it is not possible to find a lattice offset $\mathbf{x}_{0}$ that simultaneously satisfies all three equations, if the $c_{i}$ are chosen arbitrarily. Indeed, if we cross-eliminate $\mathbf{x}_{0}$ from these three equations, we obtain the following constraint on the $c_{i}$ values:

$$
\mathbf{N} \cdot \mathbf{c}=\pi\left(N_{1} P_{1} Q_{1}+N_{2} P_{2} Q_{2}+N_{3} P_{3} Q_{3}\right) \bmod 2 \pi G,
$$

where $G \equiv \operatorname{gcd}\{\mathbf{N}\}$, as before. In order to show that our condition (20) is sufficient, we need to show that it is equivalent to Eq. (A6) for every solution of Eq. (A3). That is, we need to prove the following theorem. have

Theorem. For every $\mathbf{P}, \mathbf{Q} \in \mathbb{Z}^{3}$ such that $\mathbf{P} \times \mathbf{Q}=\mathbf{N}$, we

$$
N_{1} P_{1} Q_{1}+N_{2} P_{2} Q_{2}+N_{3} P_{3} Q_{3}=N_{1} N_{2} N_{3} \quad \bmod 2 G,
$$

where $G \equiv \operatorname{gcd}\left\{\mathrm{N}_{1}, \mathrm{~N}_{2}, \mathrm{~N}_{3}\right\}$.
Proof. First, we note that for any particular solution we can construct infinitely many more solutions of the form $\mathbf{P}^{\prime}=M_{11} \mathbf{P}+M_{12} \mathbf{Q}$ and $\mathbf{Q}^{\prime}=M_{21} \mathbf{P}+M_{22} \mathbf{Q}$, where $\left(\begin{array}{ll}M_{11} & M_{12} \\ M_{21} & M_{22}\end{array}\right) \in \mathrm{SL}_{2}(\mathbb{Z})$. Since all such solutions ultimately generate the same lattice, we will regard them as equivalent. [It is also easily proved that the left-hand side of Eq. (A7) is invariant under such a transformation, modulo $2 G$, as we would expect.]

Equation (A7) is trivial in the case $\mathbf{N}=\mathbf{0}$, so we will assume, without loss of generality, that $N_{3}>0$. Because $\mathbf{P}$ and $\mathbf{Q}$ are perpendicular to $\mathbf{N}$, they must be (integer) combinations of the vectors $\left(1,0,-N_{1} / N_{3}\right)$ and $\left(0,1,-N_{2} / N_{3}\right)$. Moreover, for each solution there is a unique equivalent solution that has

$$
\begin{aligned}
\mathbf{P} & =P_{1}\left(1,0,-N_{1} / N_{3}\right)+P_{2}\left(0,1,-N_{2} / N_{3}\right) \\
\text { and } \quad & \mathbf{Q}=Q_{2}\left(0,1,-N_{2} / N_{3}\right),
\end{aligned}
$$

where $0 \leqslant P_{2}<Q_{2}$. (This simplified form is obtained by applying Euclid's algorithm to reduce the first components of $\mathbf{P}$ and $\mathbf{Q}$.) The equation $\mathbf{P} \times \mathbf{Q}=\mathbf{N}$ then becomes simply $P_{1} Q_{2}=N_{3}$, and so $P_{1}$ must be a (positive) divisor of $N_{3}$. Furthermore, the third component of $\mathbf{Q}, Q_{3}=-N_{2} / P_{1}$, must be an integer, which implies that $P_{1}$ is also a divisor of $N_{2}$. It is convenient at this point to define $H \equiv \operatorname{gcd}\left\{\mathrm{N}_{2}, \mathrm{~N}_{3}\right\}$, so that the previous two conditions can be replaced with the single condition that $P_{1}$ must be a divisor of $H$, i.e., we must have $D \equiv H / P_{1} \in \mathbb{N}$. The final requirement is that the third component of $\mathbf{P}$ must be an integer, which is equivalent to

$$
\frac{N_{1}}{D}+P_{2} \frac{N_{2}}{H}=0 \quad \bmod \frac{N_{3}}{H} .
$$

For this equation to have solutions, the first term must be an integer, and so $D$ must be a (positive) divisor of $G$. If $N_{2} \neq 0$, then $N_{2} / H$ is coprime to $N_{3} / H$, and so Eq. (A9) has exactly $D$ nonequivalent solutions for $P_{2}$. Using Euler's theorem, we can express these solutions as

$$
P_{2}=-\left(N_{1} / D\right)\left(N_{2} / H\right)^{\phi\left(N_{3} / H\right)-1}+d\left(N_{3} / H\right),
$$

where $\phi$ is the totient function and where $d=0,1, \ldots, D-$ 1 . Hence the general solution for $\mathbf{P}$ and $\mathbf{Q}$ is

$$
\begin{gathered}
\mathbf{P}=\frac{H}{D}\left(1,0,-N_{1} / N_{3}\right)+d\left(0, N_{3} / H,-N_{2} / H\right) \\
-\frac{N_{1}}{D}\left(\frac{N_{2}}{H}\right)^{\phi\left(N_{3} / H\right)-1}\left(0,1,-N_{2} / N_{3}\right) \\
\mathbf{Q}=D\left(0, N_{3} / H,-N_{2} / H\right) .
\end{gathered}
$$

So for each $D$ that is a positive divisor of $G$ we have $D$ distinct lattice solutions, indexed by $d$. The total number of distinct lattices is therefore $\sigma(G)$, i.e., the sum of all positive common divisors of $N_{1}, N_{2}, N_{3}$. In the special case where $N_{2}=0$, Eq. (A9) is automatically satisfied for any $P_{2}$, and our general solution still holds provided that we omit the final term in Eq. (A11).

Finally, we just need to verify that the general solutions (A11) and (A12) satisfy Eq. (A7), i.e., we need to prove 
that

$$
\begin{gathered}
\frac{\left(N_{2}+N_{3}\right)}{G}\left(d D \frac{N_{2} N_{3}}{H^{2}}-N_{1}\left(\frac{N_{2}}{H}\right)^{\phi\left(N_{3} / H\right)}\right) \\
=\frac{N_{1}}{G} N_{2}\left(N_{3}-1\right) \bmod 2 .
\end{gathered}
$$

We note that $\frac{N_{2}}{H} \frac{N_{3}}{H}\left(\frac{N_{2}}{H}+\frac{N_{3}}{H}\right)$ is always even, and therefore so is the first term on the left-hand side. It therefore suffices to prove that

$$
\left(N_{2}+N_{3}\right)\left(\frac{N_{2}}{H}\right)^{\phi\left(N_{3} / H\right)}=N_{2}\left(N_{3}-1\right) \bmod 2 .
$$

If $N_{2}$ is even, then both sides of this equation are even. If $N_{2}$ is odd, then both sides of this equation have the opposite parity to $N_{3}$. Therefore the equation holds in all cases.
In the special case of a $2 \mathrm{D}$ lattice, with $\mathbf{N}=(0,0, N)$, our general solution for $\mathbf{P}$ and $\mathbf{Q}$ simplifies to

$$
\mathbf{P}=(N / D, d) \text { and } \mathbf{Q}=(0, D),
$$

where $D$ is any positive divisor of $N$ and where $d=$ $0,1, \ldots, D-1$. The primitive lattice vectors are then

$$
\ell_{1}=\left(D L_{1} / N, 0\right) \quad \text { and } \quad \ell_{2}=\left(-d L_{1} / N, L_{2} / D\right)
$$

and so the sum of all $N$ lattice locations in the rectangle $\left(L_{1}, L_{2}\right)$ is

$$
\begin{aligned}
\sum_{i=0}^{(N / D)-1} \sum_{j=0}^{D-1}\left(\mathbf{x}_{0}+i \boldsymbol{\ell}_{1}+j \boldsymbol{\ell}_{2}\right) & =N \mathbf{x}_{0}+(N / 2)(N / D-1) \boldsymbol{\ell}_{1}+(N / 2)(D-1) \boldsymbol{\ell}_{2} \bmod \left(L_{1}, L_{2}\right) \\
& =N \mathbf{x}_{0}-\frac{1}{2}\left(P_{2}+Q_{2}+P_{2} Q_{2}-N\right) \mathbf{L}_{1}-\frac{1}{2}\left(P_{1}+Q_{1}+P_{1} Q_{1}-N\right) \mathbf{L}_{2} \bmod \left(L_{1}, L_{2}\right),
\end{aligned}
$$

where $\mathbf{x}_{0}$ represents any particular lattice point. From this result we can calculate the average location of the $N$ lattice points, $\overline{\mathbf{x}}$, which we used to obtain Eq. (14).

[1] N. R. Cooper, Adv. Phys. 57, 539 (2008).

[2] J. F. Annett, Superconductivity, Superfluids and Condensates, Oxford Master Series in Condensed Matter Physics, Vol. 5 (Oxford University Press, Oxford, 2004), p. 186.

[3] R. J. Donnelly, Quantized Vortices in Helium II, Cambridge Studies in Low Temperature Physics No. 3 (Cambridge University Press, Cambridge, 1991), p. 364.

[4] G. Baym, C. Pethick, and D. Pines, Nature (London) 224, 673 (1969).

[5] More generally, the circulation of canonical momentum around each vortex is $h=2 \pi \hbar$.

[6] R. P. Feynman, in Progress in Low Temperature Physics, Series in Physics, Vol. 1 (North Holland, Amsterdam, 1955), Chap. 2, pp. 17-53.

[7] W. F. Vinen, Nature (London) 181, 1524 (1958).

[8] R. J. Zieve, Y. Mukharsky, J. D. Close, J. C. Davis, and R. E. Packard, Phys. Rev. Lett. 68, 1327 (1992).

[9] G. P. Bewley, D. P. Lathrop, and K. R. Sreenivasan, Nature (London) 441, 588 (2006).

[10] M. A. Alpar, S. A. Langer, and J. A. Sauls, Astrophys. J. 282, 533 (1984).
[11] L. V. Drummond and A. Melatos, Mon. Not. R. Astron. Soc. 475, 910 (2018).

[12] A. A. Abrikosov, Zh. Eskp. Teor. Fiz. 32, 1442 (1957) [Sov. Phys. JETP 5, 1174 (1957)].

[13] E. J. Mueller and T.-L. Ho, Phys. Rev. Lett. 88, 180403 (2002).

[14] L. Mingarelli, E. E. Keaveny, and R. Barnett, J. Phys.: Condens. Matter 28, 285201 (2016).

[15] L. Mingarelli, E. E. Keaveny, and R. Barnett, Phys. Rev. A 97, 043622 (2018).

[16] M. Ruderman, T. Zhu, and K. Chen, Astrophys. J. 492, 267 (1998).

[17] V. K. Tkachenko, Zh. Eskp. Teor. Fiz. 49, 1875 (1966) [Sov. Phys. JETP 22, 1282 (1966)].

[18] S. K. Lander and D. I. Jones, Mon. Not. R. Astron. Soc. 481, 4169 (2018).

[19] W. H. Kleiner, L. M. Roth, and S. H. Autler, Phys. Rev. 133, A1226 (1964).

[20] J. S. Rutherford, Acta Crystallogr. Sect.A 48, 500 (1992).

[21] Although we have assumed a cuboidal domain, our results are easily generalized to the case of a parallelepiped with sides $\mathbf{L}_{1}, \mathbf{L}_{2}, \mathbf{L}_{3}$. In that case $P_{i}$ and $Q_{i}$ are the components of $\mathbf{p}$ and $\mathbf{q}$ in the basis that is the dual of $\left\{\mathbf{L}_{1}, \mathbf{L}_{2}, \mathbf{L}_{3}\right\}$. 